\title{
Experimental study on a new tracheal prosthesis. made from collagen-conjugated mesh
}

\begin{abstract}
A new tracheal prosthesis was made from fine Marlex mesh reinforced with a continuous polypropylene spiral. The mesh and spiral were covalently grafted and further coated with pig collagen with the aim of promoting connective tissue infiltration and providing initial airtightness. Complete surgical resection and replacement of a segment $(2 \mathrm{~cm}$ in length, three to five tracheal rings) of the cervical trachea was performed in 13 adult mongrel dogs. Two dogs died of pneumonia about 2 months after operation, and eleven dogs were killed between 3 and 26 months. The prostheses in all dogs were promptly infiltrated by the surrounding connective tissue and completely incorporated by the host trachea. Formation of respiratory epithelium, which lined the prosthetic lumen, was seen to various degrees, and, in five dogs killed at 6 months or more after reconstruction, confluent epithelization was confirmed histologically from the upper to the lower anastomotic site of the prosthesis. Marked stenosis of the prosthetic lumen caused by excessive scar tissue growth was seen in three dogs, and ulceration on the luminal surface was seen in two dogs. These results indicate that this tracheal prosthesis is highly biocompatible and promising for the repair of tracheal defects after further investigation. (J THORAC CardiovasC SuRg 1994;108:337-45)
\end{abstract}

Norihito Okumura, MD, Tatsuo Nakamura, MD, Tohru Natsume, MD, Kenji Tomihata, Yoshito Ikada, MD, PhD, and Yasuhiko Shimizu, MD, Kyoto, Japan

\begin{abstract}
$\mathrm{R}$ modifications and improvements in surgical techniques and anesthetic management have allowed tracheobronchial reconstruction to be performed actively in a clinical setting. The preferred method for tracheal reconstruction after circumferential resection is end-toend anastomosis for defects up to approximately $6 \mathrm{~cm}$ in length. ${ }^{1-7}$ However, larger tracheal defects, especially in the intrathoracic trachea, still require the use of a tracheal prosthesis. Furthermore, even within the limits of defects that can be anastomosed directly, a biocompatible prosthesis could reduce the degree of operative stress, thus expanding the surgical indications.
\end{abstract}

From Research Center for Biomedical Engineering, Kyoto University. Supported by grant-in-aid No. 01480342 for Developmental Scientific Research from the Ministry of Education, Science and Culture, Japan.

Received for publication Sept. 14, 1993.

Accepted for publication Jan. 9, 1994.

Address for reprints: Norihito Okumura, MD, Research Center for Biomedical Engineering, Kyoto University, Kawahara-cho 53, Shogoin, Sakyo-ku, Kyoto, 606, Japan.

Copyright $\stackrel{\text { c: }}{ } 1994$ by Mosby-Year Book, Inc.

$0022-5223 / 94 \$ 3.00+0 \quad \mathbf{1 2} / \mathbf{1} / \mathbf{5 4 5 2 0}$
We have been studying composites of collagen and synthetic polymers in an attempt to obtain biomaterials of high biocompatibility. ${ }^{8}{ }^{9}$ Furthermore, we have previously attempted to repair window defects of the trachea with fine Marlex mesh (C. R. Bard, Inc., Billerica, Mass.) conjugated with collagen and found that the wound healing process in the area repaired with the collagen-conjugated mesh was markedly better than that repaired with nonconjugated mesh. We also found that a $2 \times 1 \mathrm{~cm}$ defect repaired with the collagen-conjugated mesh was completely covered with normal tracheal epithelium in 6 weeks. ${ }^{10}$

Encouraged by these findings, we fabricated a novel tracheal prosthesis consisting of fine Marlex mesh (C. R. Bard) of optimal pore size, reinforced with a polypropylene spiral, and conjugated with collagen.

In this article we present the results of animal experiments with the use of this prosthesis.

\section{Materials and methods}

Collagen. Collagen was extracted from pig skin, according to the following procedure. The pig skin was chopped into small pieces and homogenized with Polytron material (Kinematika, Lucerne, Switzerland) after suspending it in cold $\mathrm{HCl}, 0.01$ $\mathrm{mol} / \mathrm{L}$, for 24 hours, followed by treatment with pepsin at $4^{\circ} \mathrm{C}$ 

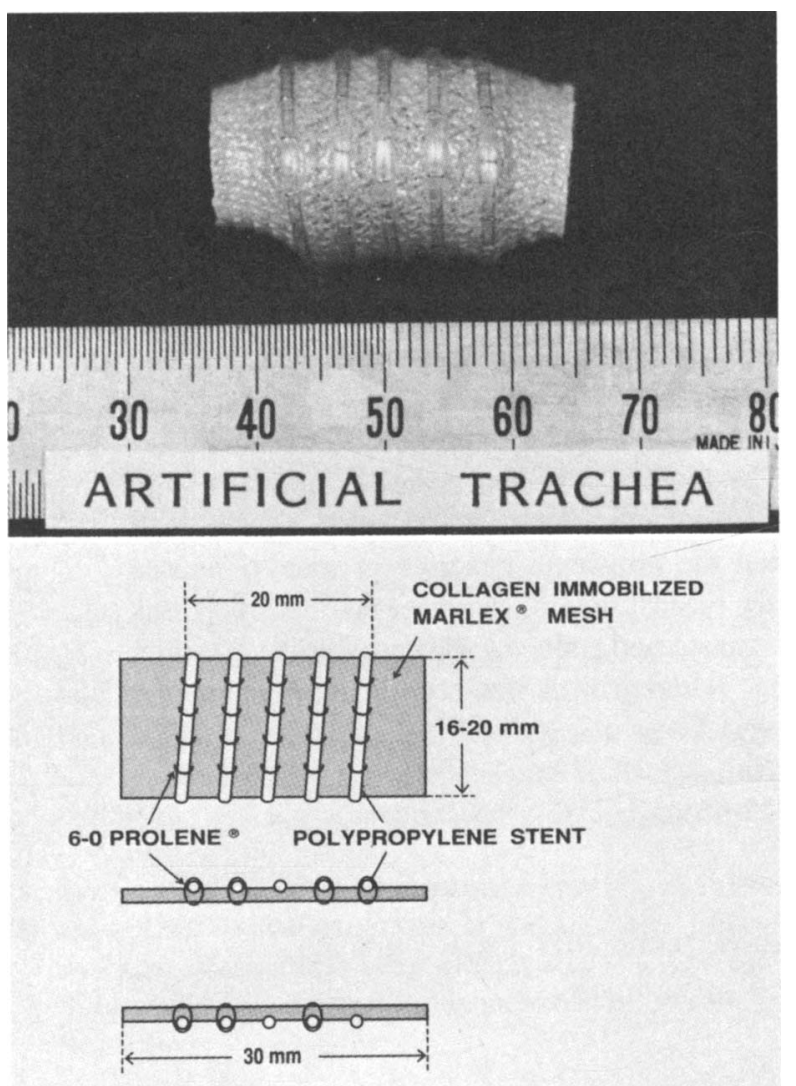

Fig. 1. Tracheal prosthesis made from collagen-conjugated mesh. For manufacturer information, see text.

for 48 hours. The resulting homogenate was centrifuged at $7000 \mathrm{~g}$ for 20 minutes, and the pellet was discarded. The supernatant consisted predominantly of type 1 collagen ( $70 \%$ to $80 \%$ ), and the rest was type 3 , which was confurmed by sodium dodecyl sulfate-polyacrylamide gel electrophoresis (SDS-PAGE). By this method, the teropeptide of collagen, which is thought to have antigenicity, was removed.

Prosthesis. The prosthesis is of cylinder type and made of fine Marlex mesh (pore size $260 \mu \mathrm{m}$, polypropylene) reinforced with a continuous polypropylene spiral, which is attached to the outside of the mesh by melting at several points and further fixed by 5-0 Prolene sutures (Ethicon, Inc., Somerville, N.J.). This procedure provides the prosthesis with a compression strength similar to that of the canine trachea. The prosthesis is $30 \mathrm{~mm}$ long with an outside diameter of 18 to $22 \mathrm{~mm}$. The polypropylene spiral is attached to the cylinder along a $20 \mathrm{~mm}$ segment. The prosthesis is covalently immobilized and then further physically coated with collagen to promote host tissue incorporation and render the prosthesis airtight during the initial stage of implantation (Fig. 1).

The method used for fabricating the collagen-prosthesis composite was as follows. The prosthesis was first exposed to a plasma discharge to introduce peroxides onto the surface so that graft polymerization of acrylic acid on the cylinder surface could be performed. The oxidized surface was covalently immobilized with collagen by immersion in $1 \% \mathrm{w} / \mathrm{v}$ collagen solution of ace- (a) Plasma discharge

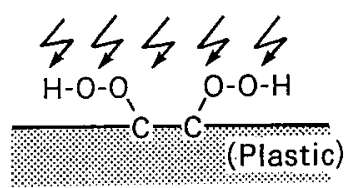

(b) Graft polymerization of acrylic acid

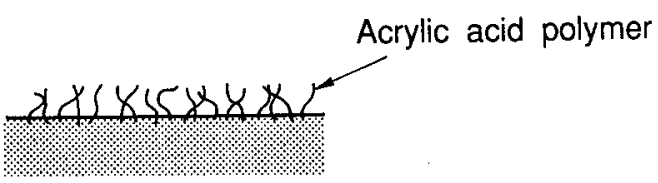

(c) Collagen coupling

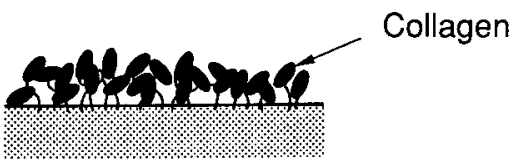

(d) Collagen coating

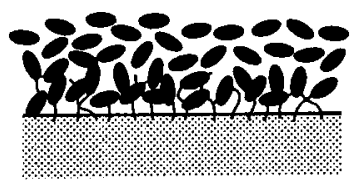

(e) UV - irradiation

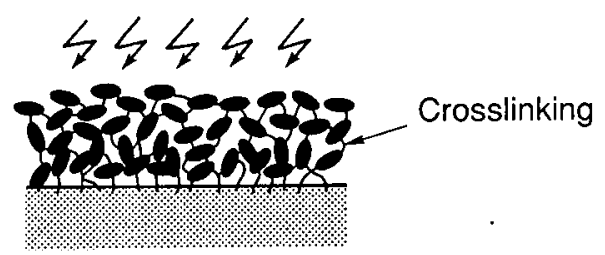

Fig. 2. Fabricating method of collagen-plastic composite. $U V$, Ultraviolet.

tic acid, $1 \mathrm{mmol} / \mathrm{L}$, after activation with a water-soluble carbodiimide. The prosthesis was then coated thickly with collagen and then cross-linked by ultraviolet light (Fig. 2). ${ }^{11}$ The irradiation was performed in atmospheric air at $15 \mathrm{~W}$ at a distance of $50 \mathrm{~cm}$ from the ultraviolet lamp source for 16 hours. The total weight of the collagen conjugated to the prosthesis was about 0.8 gm.

Animal experiment. Thirteen adult mongrel dogs, weighing 8 to $15 \mathrm{~kg}$, were used. Anesthesia was induced with an intramuscular injection of ketamine hydrochloride, $10 \mathrm{mg} / \mathrm{kg}$ body weight, and xylazine, $2 \mathrm{mg} / \mathrm{kg}$ body weight, and then maintained by intravenous injection of sodium pentobarbital, 25 $\mathrm{mg} / \mathrm{kg}$ body weight. Spontaneous respiration was maintained.

The cervical trachea was exposed through a midline incision in the neck. Circumferential defects, each $2 \mathrm{~cm}$ long, including 

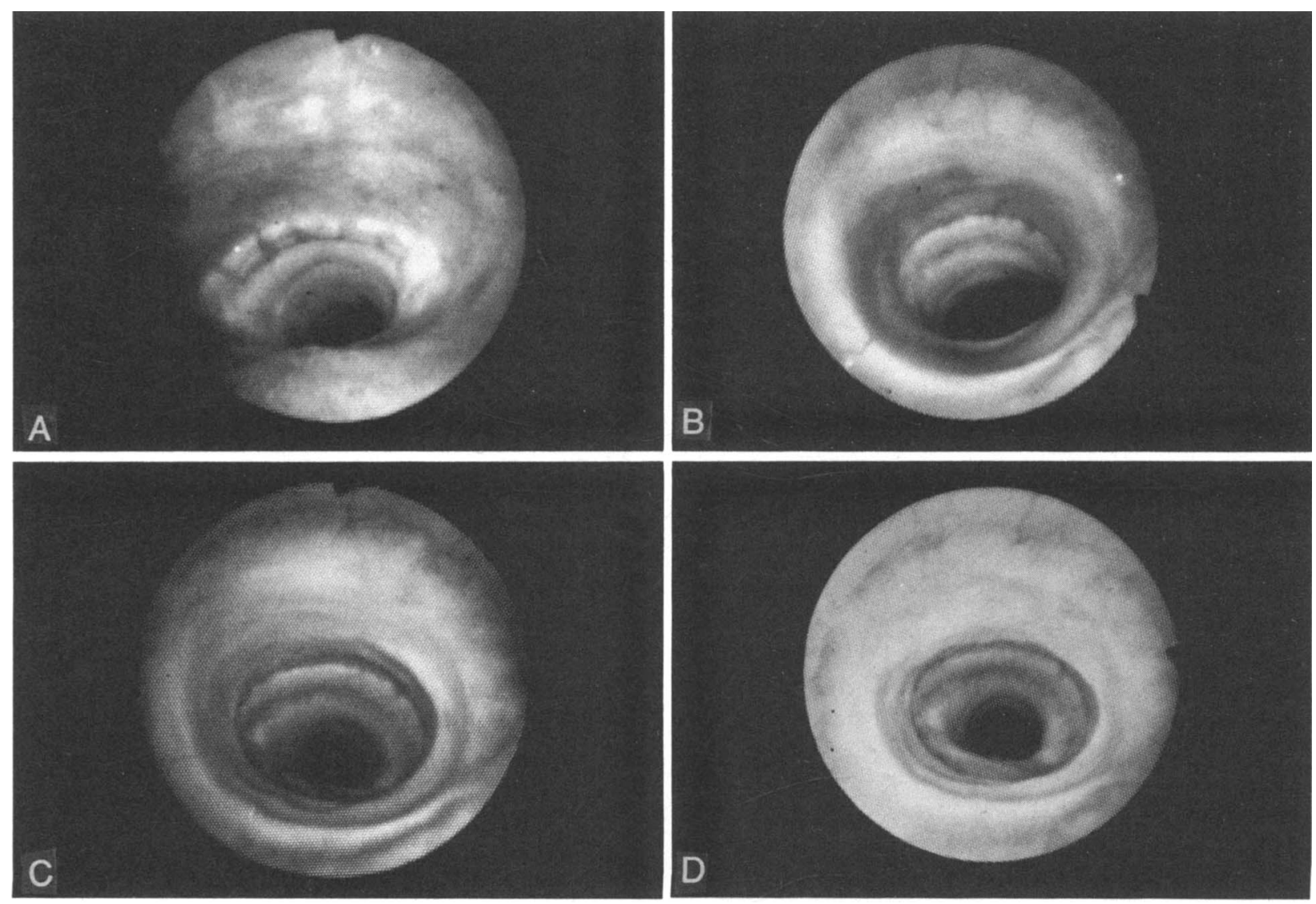

Fig. 3. Photographs taken through fiberoptic bronchoscope. A, One month after operation; prosthesis has been already infiltrated by host connective tissue. B, Three months after operation; luminal surface of prosthesis appears partially lustrous, indicating mucosal maturation. C, Six months after operation; whole luminal surface seems smooth and lustrous. D, Twenty-four months after operation; color and luster of prosthetic lumen are similar to those of native trachea, with minimal luminal stenosis.

three to five tracheal cartilages, were created in the lower cervical trachea. The tracheal stumps, including one tracheal ring, were inserted within the prosthesis and anastomosed with 3-0 Vicryl sutures (Ethicon). The soft tissues were then closed in layers. As a rule, $1 \mathrm{gm}$ of ampicillin was given intravenously on the day of operation, and then $500 \mathrm{mg}$ by mouth was given daily after the operation for 1 month.

Bronchoscopic examination was performed periodically under general anesthesia. The animals were killed at intervals of 3 to 24 months. The trachea was resected en bloc, and gross examination was conducted. Histologic study was performed by both light microscopy after staining with hematoxylin and eosin and scanning electron microscopy.

All operations and euthanasia were performed in accordance with the Animal Welfare Regulation Guidelines of Animal Experimentation Committee of Kyoto University (1989).

\section{Results}

Examination of the tracheal prosthesis at 3 weeks after operation with a fiberoptic bronchoscope showed that the prosthesis had already been infiltrated by the host connective tissue, rendering the mesh unrecognizable. By 2 months, the luminal surface of the prosthesis appeared partially lustrous, indicating mucosal maturation. No marked stenosis was seen at the prosthetic lumen at this stage (Fig. 3).

After 3 months, the luminal surface of the prosthesis became similar to that of the host trachea. Stenosis of the prosthetic lumen was observed in some dogs, although generally mild, but it became more severe with time in three dogs.

Two dogs died of pneumonia about 2 months after operation. They were free from any anastomotic dehiscence, severe luminal stenosis, or massive infection of the prosthesis, which might have been considered to have been related to their deaths. The other 11 dogs were killed between 3 and 26 months after operation (Table I), and two of them were killed at $4 \frac{1}{2}$ and $171 / 2$ months after 


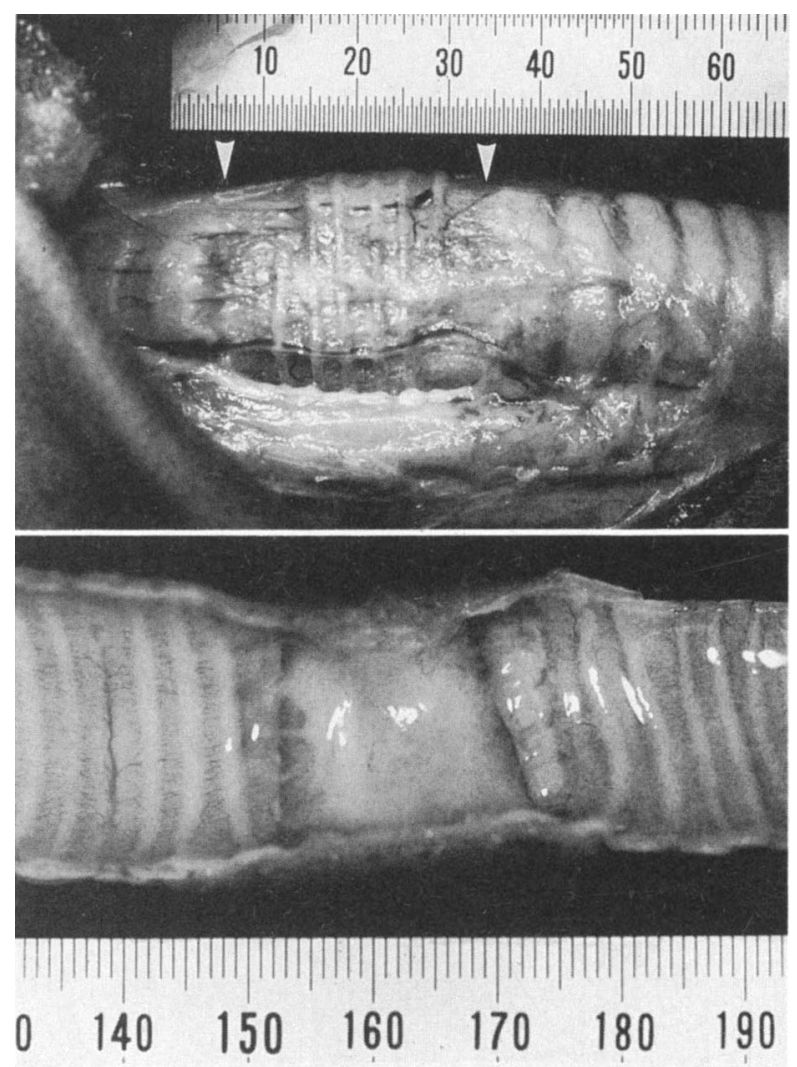

Fig. 4. Macroscopic views of the reconstructed trachea 6 months after operation (dog No. 6). Top, External view; prosthesis (between arrows) is completely incorporated into host and retains its original shape by means of polypropylene spiral, which acts as tracheal cartilage. Bottom, Internal view; inner surface is covered with smooth and lustrous soft tissue similar to that of native trachea.

operation because of severe stridor. The other nine dogs were without symptoms at the time they were killed.

In all dogs surviving longer than 2 months after operation, the prostheses were entirely infiltrated by host connective tissue and completely incorporated by the body. No damage to the local surrounding tissues was observed. In most cases, the polypropylene spirals acted as tracheal cartilage, retaining the original shape of the prostheses (Fig. 4). In five dogs, stenosis occurred in the prosthetic lumen, mostly in the midportion of each prosthesis. Necropsy showed that the stenosis was a result of excessive scar tissue growth and mild deformation of the prosthesis. In three dogs, partial exposure of the mesh on the luminal surface (ulceration) was observed.

Histologic examination showed that the connective tissue, including vessels, had invaded the mesh pores, covering the inner surface of the prosthesis in all the dogs.
Also in these animals, neoepithelium appeared stretched over the connective tissue layer from the cut end of the tracheal stump (Fig. 5). Coated collagen filling the mesh pores was already unrecognizable in dogs that died 2 months after operation.

Formation of respiratory epithelium from the upper to the lower anastomotic site of the prosthesis was confirmed histologically in five dogs surviving for more than 6 months. This epithelium consisted of pseudostratified ciliated columnar epithelium over a large area of the reconstruction, but squamous epithelial cells were partially evident in the midportion of the prosthesis (Fig. 6). The ciliated columnar epithelium rested on an abundantly vascularized loose connective tissue layer, reminiscent of the tunica propria of the natural trachea, with interposition of the basement membrane. On the other hand, there was only a dense fibrous tissue layer below the squamous epithelium, and neither basement membrane nor loose connective tissue layer was observed (Fig. 6). Ingrowth of the dense fibrous tissue layer was remarkable in the dogs with luminal stenosis in the center of the prosthesis. Epithelial lining was disrupted in the tracheal luminas that were strongly stenosed.

Observation by scanning electron microscopy showed long and uniform cilia on the luminal surface over a large area, gathering closely together. However, in the midportion of the prosthesis, nonuniform cilia were scattered and microvilli were evident around them (Fig. 7).

\section{Discussion}

Studies on the prostheses for repairing circumferential tracheal defects have a long history, beginning with Daniel's experiment in $1948 .{ }^{12}$ Since then, a great variety of tubular prostheses have been used, but, without exception, the results have been unsatisfactory in a significant proportion of cases.

One of the main reasons that prosthetic tracheal reconstruction is difficult is that the trachea is located at a site facing the "external environment." Therefore, the implanted tracheal prosthesis continues to exist between the external environment and the inner body, as long as the prosthesis is not incorporated by the host. Accordingly, the wound healing process, which tries to absorb the foreign substance into the external environment, persists at the implantation site. This process results in overgrowth of granulation tissue or in rejection of the prosthesis after implantation. Infection, which inevitably occurs on the part of the prosthesis facing the external environment, also makes prosthetic tracheal reconstruction difficult.

In view of these problems, porous materials that can be incorporated by the host tissue seem to be more suitable for tracheal reconstruction than nonporous materials, 


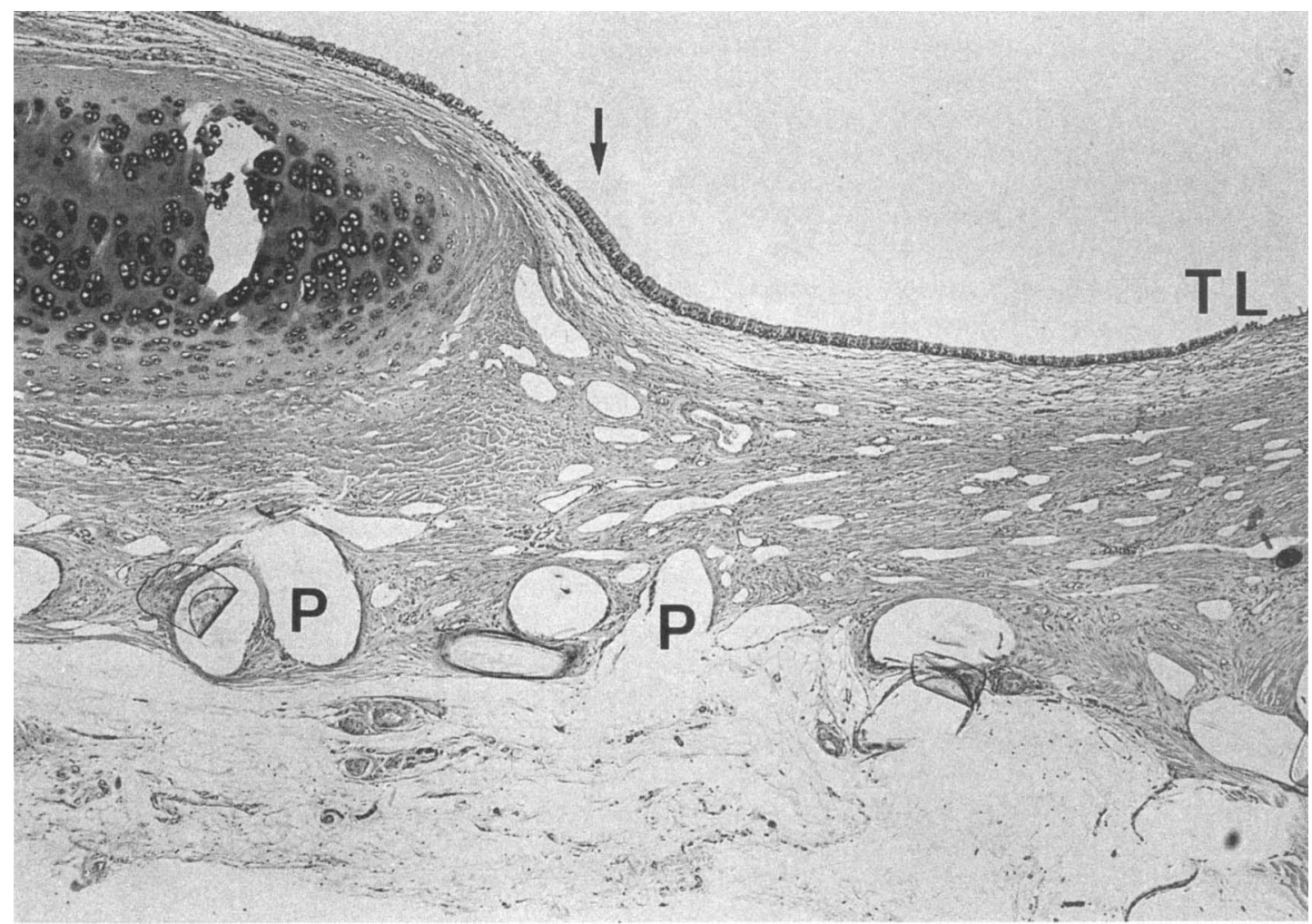

Fig. 5. Photomicrograph of longitudinal section taken from prosthesis 21 months after operation (dog No. 8). Continuous cover of respiratory epithelium overlying a thin layer of connective tissue containing small vessels is seen (hematoxylin and eosin stain, original magnification $\times 40$ ). Arrow shows anastomotic site; $T L$, tracheal lumen; $P$, prosthesis.

Table I. Experimental results of tracheal reconstruction with the prosthesis

\begin{tabular}{|c|c|c|c|c|c|c|c|}
\hline \multirow[b]{2}{*}{ Dog No. } & \multirow{2}{*}{$\begin{array}{c}\text { Removed } \\
\text { rings }\end{array}$} & \multicolumn{2}{|c|}{ Survival (mo) } & \multirow[b]{2}{*}{ Dehiscence } & \multirow[b]{2}{*}{ Stenosis* } & \multirow[b]{2}{*}{ Ulceration } & \multirow[b]{2}{*}{ Epithelialization } \\
\hline & & Dead & Killed & & & & \\
\hline 1 & 5 & 2 (pneumonia) & - & - & - & - & Trace \\
\hline 2 & 5 & 2 (pneumonia) & - & - & + & - & Trace \\
\hline 3 & 4 & & 26 & - & - & - & Confluent \\
\hline 4 & 3 & & 24 & - & - & - & Confluent \\
\hline 5 & 4 & & 12 & - & ++ & - & Partial \\
\hline 6 & 3 & & 6 & - & - & - & Confluent \\
\hline 7 & 3 & & 22 & - & - & + & Partial \\
\hline 8 & 4 & & 21 & - & + & - & Confluent \\
\hline 9 & 4 & & 19 & - & - & + & Trace \\
\hline 10 & 4 & & 17.5 & - & ++ & - & Partial \\
\hline 11 & 5 & & 4.5 & - & ++ & - & Partial \\
\hline 12 & 4 & & 3 & - & - & - & Partial \\
\hline 13 & 4 & & 26 & - & - & + & Confluent \\
\hline
\end{tabular}

* Reduction in tracheal lumen: - , Less than one third of native tracheal diameter;,+ from one third to two thirds of native tracheal diameter; ++ , more than two thirds of native tracheal diameter.

which are continuously exposed to the external environment (i.e., the tracheal lumen).

In the past, many types of porous synthetic materials were used for tracheal reconstruction. ${ }^{13-20}$ Heavy Marlex mesh (polyethylene) was also applied clinically by some investigators with some degree of success. ${ }^{15}$ However, this material was later abandoned because its application was proved to be associated with rupture of the innominate 

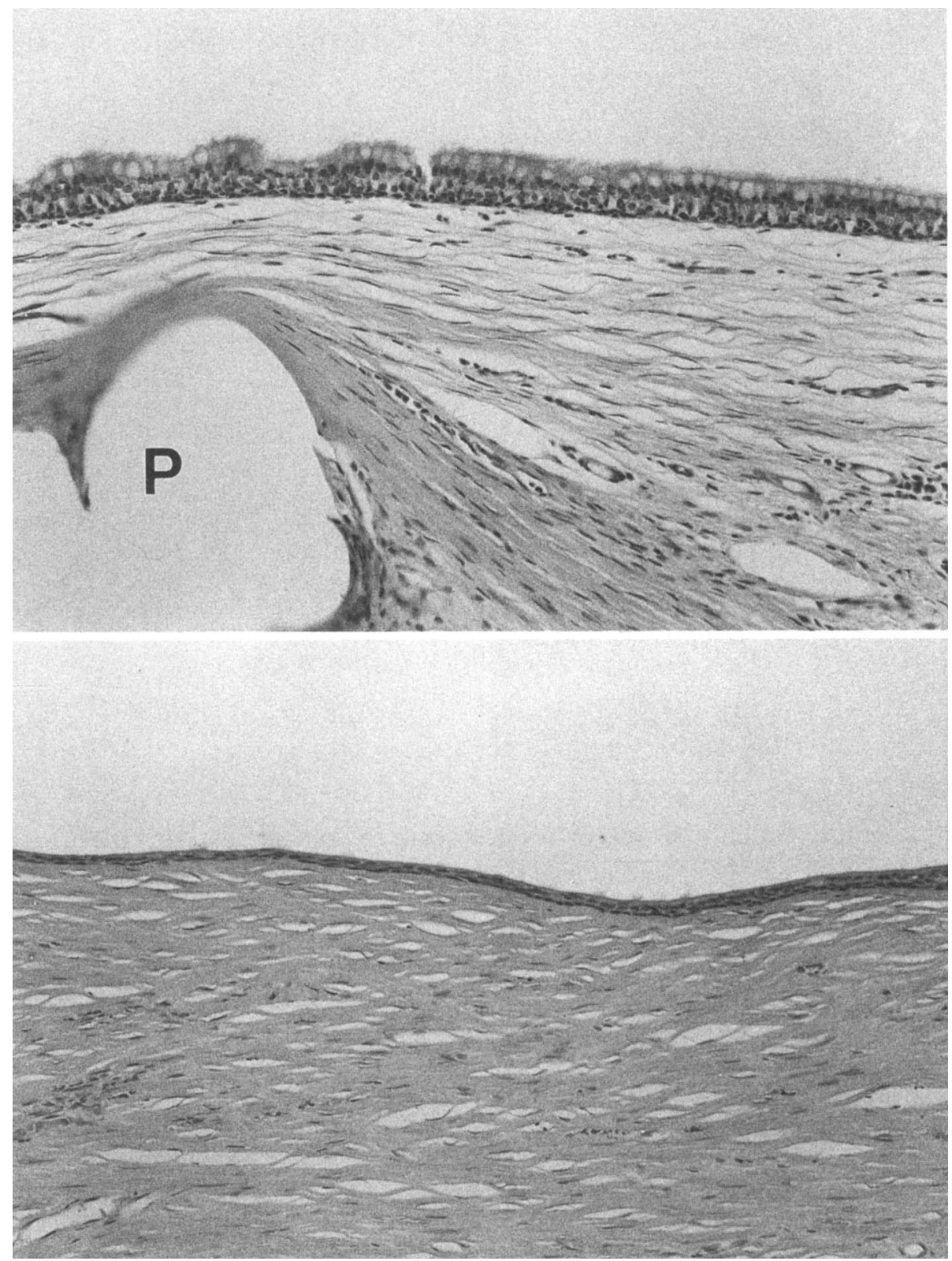

Fig. 6. High-power microscopic view taken from the lining of the prosthesis 6 months after operation (dog No. 6). Top, Pseudostratified columnar ciliated epithelium with basement membrane covers prosthetic lumen. Bottom, Midportion of the prosthesis is covered with layer of stratified squamous epithelium (hematoxylin and eosin stain, original magnification $\times 200$ ). $P$, Prosthesis.

artery, which was usually fatal. ${ }^{21}$ These problems are thought to be closely related to the pore size and stiffness of the mesh.

In our previous study, we found that the optimal pore size of the mesh for tracheal prosthesis, with regard to the wound healing process, is about $300 \mu \mathrm{m} .{ }^{22}$ Furthermore, in our laboratory, we have been investigating composites of collagen and a synthetic polymer in an attempt to obtain biomaterials of high biocompatibility. Collagen, a natural polymer, possesses numerous desirable features as a biomaterial, including controllable biodegradation, promotion of cellular growth and attachment, low antigenicity, minimal inflammation, and noncytotoxicity ${ }^{23-26}$ Previously, we performed repair of window defects in 

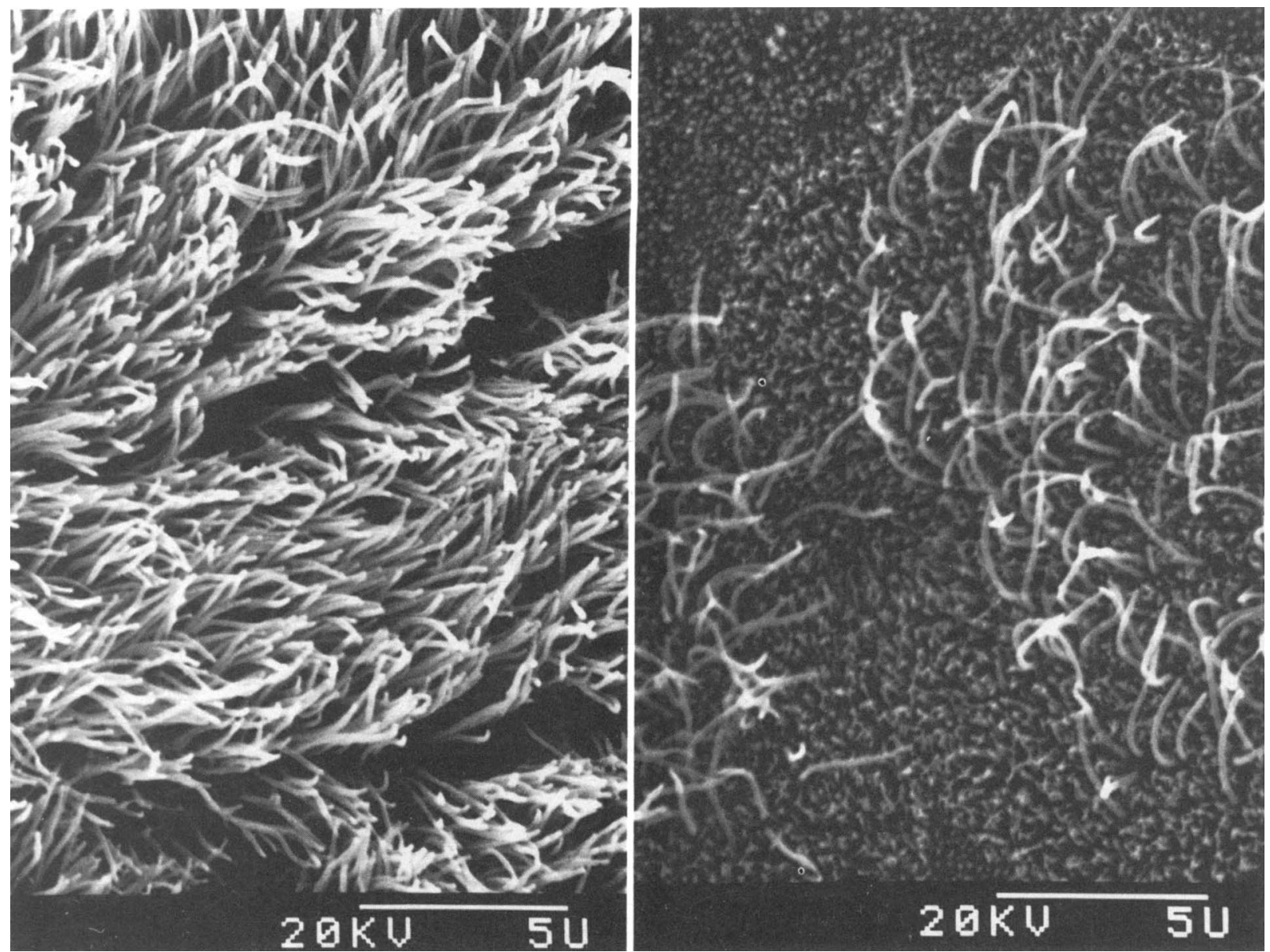

Fig. 7. Scanning electron photomicrographs taken from inner surface of prosthesis 6 months after operation (dog No. 6). Left, Marginal portion close to the anastomotic site; long and uniform cilia grow thick. Right, Midportion of the prosthesis; nonuniform cilia are scattered, and microvilli are evident around them.

tracheas with the use of fine Marlex mesh conjugated with collagen and found that the wound healing process in the area repaired with the collagen-conjugated mesh was markedly better than that with the mesh without collagen. ${ }^{10}$

In the present study, for the replacement of circumferential tracheal defects, we fabricated a tracheal prosthesis $3 \mathrm{~cm}$ in length from fine Marlex mesh reinforced with a polypropylene spiral on which collagen was chemically bound and further physically coated. The short length (3 $\mathrm{cm}$ ) of this prosthesis may prompt general doubts about the propriety of its clinical application. However, the practical value of current tracheal prosthesis consists in their role to fill the gap between the actual length of the resected trachea and a length that allows end-to-end anastomosis. Many prior studies have demonstrated that direct anastomosis is possible for tracheal defects up to about $6 \mathrm{~cm} \cdot{ }^{1-7}$ Therefore, the resected trachea does not have to be replaced by a tracheal prosthesis of the same length. From this viewpoint, we do not claim, of course, that reconstruction with prostheses $3 \mathrm{~cm}$ in length solves all the problems, but there seems to be little need to use much longer tracheal prostheses in the actual clinical setting.

In the present experiments, no anastomotic problems such as dehiscence and overgrowth of granulation tissue occurred, which suggests that our tracheal prosthesis was more biocompatible than were any other synthetic prosthetic grafts tested thus far. This absence of problems is clearly due to our use of a mesh of optimal pore size conjugated with collagen. Moreover, the light ultraviolet cross-linking in the collagen without chemical modifiers may have worked in favor of the tissues. The mechanical and structural properties of our prosthesis, which are close to those of the natural organ, also seem to have contributed to the good results. Fine Marlex mesh is thin and soft, 
and the supporting strength of the prosthetic lumen is imposed on the polypropylene spiral attached to the mesh. This structural design makes our tracheal prosthesis not only resistant to local mechanical stress, but also flexible enough to allow some movement of the vital contiguous structures. Because of these properties, neither damage to local surrounding tissues nor problems with the anastomosis were seen.

Luminal stenosis was among the problems encountered, occurring in some replacements. Histologically, overgrowth of scar tissue was observed at all the stenotic sites, and the epithelial lining over the thick scar tissue was always disrupted. However, overgrowth of scar tissue was seldom seen in the dogs where epithelization was found to be confluent throughout the length of the prosthesis. These findings suggest that proliferation of granulation tissue, which is a process of wound healing, persists as long as the luminal surface is not covered by epithelium; however, once the surface is covered by epithelium, the process of stenosis slows down or stops. Deformation of the prosthesis was seen in some of the reconstructions which were complicated by luminal stenosis. However, this deformation is probably due to the use of dogs as experimental animals because canine neck mobility is much more extensive than that of human beings.

As for epithelization, confluent epithelial coverage throughout the prosthetic lumen was confirmed histologically in five dogs which were killed 6 months or more after operation. This result seemed better than that achieved in previous studies. ${ }^{16-21}$ However, in the other dogs that showed incomplete prosthetic epithelization, only squamous or cuboidal epithelial cells were seen near the anastomotic site. These findings suggest that the epithelium in the prosthesis is formed as a result of progressive transformation, as some investigators have already reported..$^{10,17}$ According to these results, columnar epithelial cells at the cut edge of the native trachea lost their columnar orientation and began to show squamous multilayered stratification. This process was followed by migration of squamous, poorly differentiated cells, which became progressively cuboidal, polarizing themselves so that they lay with their long axis perpendicular to the surface and eventually differentiating to ciliated columnar epithelium. In four dogs, the epithelial lining of the prosthetic lumen was discontinuous even after 6 months after the operation and inflammatory cell infiltration was evident near the surface where epithelium was disrupted. These observations imply that asymptomatic local infection may have prevented the process of epithelization. Therefore, reducing the chances of local infection at the prosthetic lumen seems to be one of the most important ways of obtaining complete epithelization and may help to prevent luminal stenosis, which is a major complication of prosthesis application.

So far, most of the porous tracheal prostheses, reported previously with limited success, have been used in combination with autogenous materials ${ }^{16}$ or by means of a staged approach, which involves burying the prosthesis in a subcutaneous pocket before tracheal resection ${ }^{18}$ to provide air-sealing and tissue affinity for the prosthesis. In the present experiment, however, we performed tracheal replacement with our new prosthesis without these complicated procedures and obtained successful results that are not at all inferior to those of other previous studies. These results also confirm the high biocompatibility of our current prosthesis.

Therefore, we conclude that, with further improvements in promoting complete epithelization of the lumen, our tracheal prosthesis is apparently promising for the repair of tracheal defects.

\section{REFER EN CES}

1. Grillo HC. Reconstruction of the trachea: experiences in 100 consecutive cases. Thorax 1973;28:667-79.

2. Grillo HC. Surgical treatment of postintubation tracheal injuries. J Thorac CARDIOvasC SURG 1979;78:860-75.

3. Grillo HC. Primary reconstruction of airway after resection of subglottic laryngeal and upper tracheal stenosis. Ann Thorac Surg 1982;33:3-18.

4. Grillo HC, Zannini P, Michelassi F. Complications of tracheal reconstruction; incidence, treatment, and. prevention. J ThORAC CARDIOVASC SURG 1986;91:322-8.

5. Grillo HC, Dignan EF, Miura T. Extensive resection and reconstruction of mediastinal trachea without prosthesis or graft: an anatomical study in man. J THORAC CARDIOvASC SURG 1964;48:741-9.

6. Grillo HC. Circumferential resection and reconstruction of the mediastinal and cervical trachea. Ann. Surg 1965; $162: 374-88$.

7. Mulliken JB, Grillo HC. The limits of tracheal resection with primary anastomosis: further anatomical studies in man. J THORAC CARdiovasc Surg 1968;55:418-21.

8. Shimizu Y, Abe R, Teramatsu T. Studies on copolymers of collagen and a synthetic polymer: first report-experimental study on biocompatibility of laminar copolymers of collagen and a synthetic polymer. Biomat Med Dev Art Org 1977;5:49-66.

9. Shimizu Y, Miyamoto Y, Teramatsu T. Studies on composites of collagen and a synthetic polymer: second reportmode of reaction of a laminar composite with living tissue, and results of long-term implantation. Biomat Med Dev Artif Organs 1978;6:375-91.

10. Hirai K, Shimizu Y, Hino T. Epithelial regeneration in collagen-coated and uncoated patch grafts implanted into dog tracheas. J Exp Pathol 1990;71:51-62.

11. Okada T, Tamada Y, Ikada Y. Surface modification of sil- 
icone for tissue adhesion. In: Pizzoferato A, ed. Biomaterials and clinical application. Amsterdam: Elsevier Science Publishers, 1987:465-70.

12. Daniel RA Jr. The regeneration of defects of the trachea and bronchi: an experimental study. J THORAC SURG 1948;17:335-49.

13. Bucher RM, Burnett WE, Rosemond GP. Experimental reconstruction of the tracheal and bronchial defects with stainless steel wire mesh. J THORAC SURG 1951;21:57283.

14. Beall AC Jr, Harrington OB, Greenberg SD, Morris GC $\mathrm{Jr}$, Usher FC. Tracheal replacement with heavy Marlex mesh. Arch Surg 1962;84:390-6.

15. Pearson FG, Henderson RD, Gross AE, Ginsberg RJ, Stone RM. The reconstruction of circumferential tracheal defects with a porous prosthesis: an experimental and clinical study using heavy Marlex mesh. J THORAC CARDIOVASC SURG 1968;55:605-16.

16. Nelson RJ, Goldberg L, White RA, Shors E, Hirose FM. Neovascularity of a tracheal prosthesis/tissue complex. J Thorac Cardiovasc Surg 1983;86:800-8.

17. Leake D, Habal M. Prosthetic replacement of large defects of the cervical trachea in dogs. Biomaterials 1985;6; $17-22$.
18. Jacobs JR. Investigations into tracheal prosthetic reconstruction. Laryngoscope 1988;98:1239-45.

19. Schauwecker HH, Gerlach J, Planck H, Bucherl ES. Isoelastic polyurethane prosthesis for segmental tracheal replacement in beagle dogs. Artif Organs 1989;13:216-8.

20. Jorge RG, Ramos AS, de Guevara ACL, Huedo FM, de Vega MG. Experimental study of a new porous tracheal prosthesis. Ann Thorac Surg 1990;50:281-7.

21. Deslauriens J, Ginsberg RJ, Nelems JM, Pearson FG. Innominate artery rupture: a major complication of tracheal surgery. Ann Thorac Surg 1975;20:671-7.

22. Shimizu $Y$, Tamura $K$, Kato $H$, Teramatsu $T$, Hino $T$. Study of the artificial trachea using mesh. Jpn J Artif Organs 1983;12:486-9.

23. Elsdale T, Bard J. Collagen substrata for studies on cell behavior. J Cell Biol 1972;54:626-37.

24. Schor SL. Cell proliferation and migration on collagen substrata in vitro. J Cell Sci 1980;41:159-75.

25. Ruoslahti E, Hayman EG, Pierschbacher MD. Extracellular matrices and cell adhesion. Arteriosclerosis 1985;5:58194.

26. Mian M, Beghe F, Mian E. Collagen as a pharmacological approach in wound healing. Int $\mathbf{J}$ Tiss Reac 1992;14(Suppl):1-9. 